\title{
Rare events, exponential hitting times and extremal indices via spectral perturbation
}

\author{
Gerhard Keller
}

December 22, 2011

\begin{abstract}
We discuss how an eigenvalue perturbation formula for transfer operators of dynamical systems is related to exponential hitting time distributions and extreme value theory for processes generated by chaotic dynamical systems. We also list a number of piecewise expanding systems to which this general theory applies and discuss the prospects to apply this theory to some classes of piecewise hyperbolic systems.
\end{abstract}

\section{Introduction}

The first occurence of a rare event $A_{\varepsilon}$ in a time discrete dynamical system $T: M \rightarrow M$ can be described in terms of the first hitting time $\tau_{\varepsilon}(x)=\inf \left\{i \geqslant 0: T^{i} x \in A_{\varepsilon}\right\}(x \in M)$. In order to justify the adjective "rare" one should assume that $\mu\left(A_{\varepsilon}\right)$ is small, where $\mu$ is a suitable reference probability measure on $M$. For many dynamical systems it is then known that the law of $\tau_{\varepsilon}$ under $\mu$ is nearly exponential with a parameter $\lambda_{\varepsilon}$ that is closely related to $\mu\left(A_{\varepsilon}\right)$, see [18] for an excellent review. In this note we describe how asymptotic expressions (as $\varepsilon \rightarrow 0$ ) for the exponent $\lambda_{\varepsilon}$ of exponential hitting time statistics can be derived from spectral perturbation results for the Perron Frobenius operator of the dynamical system, provided the operator has a spectral gap on a suitable space of (generalized) functions.

\subsection{Perturbation theory for dynamical systems}

In 1999, C. Liverani and the author 41 published a spectral perturbation theorem for linear operators that is particularly suited to deal with transfer operators of dynamical systems: it does not require the perturbation to be small in operator norm (which is rarely the case for transfer operators if the underlying dynamics are perturbed). Of course there is a price to pay for this: the operators must satisfy a uniform inequality which is known as Lasota-Yorke inequality in dynamical systems or as Doeblin-Fortet inequality in the theory of Markov operators, and the conclusions are not as sharp as one might expect, namely the perturbed spectral quantities do not depend Lipschitz-continuously on the size of the perturbation.

There are in fact examples showing that the latter drawback of this perturbation theorem is not just an artifact of the proof [8, Theorem 6.1 and Footnote 7]. Therefore, a perturbation result for the leading eigenvalue of transfer operators published by C. Liverani and the author [44 in 2009 might seem contradictory at first sight: under rather general assumptions that are met in particular when the aforementioned spectral perturbation theorem applies, this result shows that the leading eigenvalue of a transfer operator is differentiable as a function of the size of the perturbation at the unperturbed operator. This covers the case where the leading eigenvalue becomes smaller than 1 due to a small leak in the phase space of the system so that this eigenvalue determines the escape rate of the open system. 


\subsection{Escape rates in open dynamical systems}

The study of escape rates (also called decay rates) in open dynamical systems has different aspects. Starting with 50, 49 there are numerous publications highlighting the role these rates play for conditionally invariant measures, see [18, 23] for reviews and further perspectives. In [39] and [40, section $9.6 \mathrm{C}]$ escape rates were characterized in terms of topological pressure, and [22] is a recent systematic account of this approach.

Here, on the contrary, the focus is on precise approximations of the escape rate for small holes. This work was motivated by a result of Bunimovich and Yurchenko [17, Theorem 4.6.1] that I first learned about during a conference at the Erwin-Schrödinger Institute in 2008. It gave rise to the paper [44. Since that paper was written, not only several other authors considered related problems on escape rates and intermittent phenomena [4, 5, 6, 22, 24, 28, 29, but I also had the opportunity to learn about the close relations between escape rates, hitting and return time statistics and extremal indices for extreme value distribution 1$]$ [25, 27, 26]. In particular the extremal index turns out to be a quantity that appears also in the eigenvalue perturbation formula from [44, and the sharp error term for exponential hitting times that was proved for mixing processes in [1] is also closely related to the same formula.

\subsection{Outline of this paper}

The goal of this note is to provide details for the interrelations described above in the particular case where the dynamics of open systems with small holes are described by a perturbed Perron Frobenius operator. In particular,

- some details are given why the approximation formula for the leading eigenvalue is valid in the perturbative setting from [4] (section 2),

- a slight extension of the arguments from [44] is given that provides estimates for the error term in the exponential approximation of the distribution of hitting times (section 3.2),

- it is argued that exponential hitting time statistics (including a formula for the extremal index) follow from the formula for the leading eigenvalue (section 3.3),

- and a number of concrete settings are listed to which the perturbation formula applies (section (4).

\section{A review of some perturbation results}

The paper [44] provides a first order approximation (as $\varepsilon \rightarrow 0$ ) for the leading eigenvalues of the Perron-Frobenius operator of an open dynamical system with a hole of "size" $\varepsilon$. The main result is formulated as a perturbation theorem for the leading eigenvalue of a family $\left(P_{\varepsilon}\right)_{\varepsilon \in E}$ of linear operators on a Banach space $(V,\|\|$.$) , where E$ is a set of parameters (equipped with some topology) containing a parameter called 0 such that $P_{0}$ describes the dynamics without a hole. This theorem applies whenever the following assumptions (11) - (6) can be verified:

There are $\lambda_{\varepsilon} \in \mathbb{C}, \varphi_{\varepsilon} \in V$, a bounded linear functional $\nu_{\varepsilon}: V \rightarrow \mathbb{C}$, and linear operators $Q_{\varepsilon}: V \rightarrow V$ such that

$$
\begin{gathered}
\lambda_{\varepsilon}^{-1} P_{\varepsilon}=\varphi_{\varepsilon} \otimes \nu_{\varepsilon}+Q_{\varepsilon}\left(\text { where we assume } \lambda_{0}=1\right), \\
P_{\varepsilon}\left(\varphi_{\varepsilon}\right)=\lambda_{\varepsilon} \varphi_{\varepsilon}, \quad \nu_{\varepsilon} P_{\varepsilon}=\lambda_{\varepsilon} \nu_{\varepsilon}, \quad Q_{\varepsilon}\left(\varphi_{\varepsilon}\right)=0, \quad \nu_{\varepsilon} Q_{\varepsilon}=0, \\
\sum_{n=0}^{\infty} \sup _{\varepsilon \in E}\left\|Q_{\varepsilon}^{n}\right\|=: C_{1}<\infty,
\end{gathered}
$$

\footnotetext{
${ }^{1} \mathrm{I}$ want to mention in particular the talk by J. Freitas "Laws of rare events for chaotic dynamical systems" and stimulating conversations with L. Bunimovich and M. Demers at the conference "Large deviations in dynamical systems" at the CIRM in June 2011.
} 
Observe that assumptions (11) and (2) imply $\nu_{\varepsilon}\left(\varphi_{\varepsilon}\right)=1$ for all $\varepsilon 2$ The fourth assumption relates the "size" of $\varphi_{\varepsilon}$ to that of $\varphi_{0}$ :

$$
\exists C_{2}>0 \forall \varepsilon \in E: \nu_{0}\left(\varphi_{\varepsilon}\right)=1 \text { and }\left\|\varphi_{\varepsilon}\right\| \leqslant C_{2}<\infty .
$$

Now let

$$
\Delta_{\varepsilon}:=\nu_{0}\left(P_{0}-P_{\varepsilon}\right)\left(\varphi_{0}\right)
$$

where $\nu_{0}\left(P_{0}-P_{\epsilon}\right)$ denotes the composition $\nu_{0} \circ\left(P_{0}-P_{\epsilon}\right): V \rightarrow \mathbb{C}$. Then the last two assumptions are

$$
\begin{gathered}
\lim _{\varepsilon \rightarrow 0}\left\|\nu_{0}\left(P_{0}-P_{\varepsilon}\right)\right\|=0, \\
\left\|\nu_{0}\left(P_{0}-P_{\varepsilon}\right)\right\| \cdot\left\|\left(P_{0}-P_{\varepsilon}\right) \varphi_{0}\right\| \leqslant \text { const } \cdot\left|\Delta_{\varepsilon}\right|
\end{gathered}
$$

Under these assumptions the main result of [44] is the formula

$$
1-\lambda_{\varepsilon}=\Delta_{\varepsilon} \theta(1+o(1)) \text { in the limit } \varepsilon \rightarrow 0
$$

where $\theta$ is a constant that takes care of short time correlations, see section 3.1 for more details.

\section{$2.1 \quad$ Verifying assumptions (1) - (4)}

One way to verify assumptions (11) - (4) that was already taken in [44] and [24], is to use the spectral perturbation theorem from [41. It holds (not only) under the following assumptions:

i. There are constants $\alpha \in(0,1), D>0$ and a second norm $|\cdot|_{w} \leqslant\|\cdot\|$ on $V$ (it is enough that this is a seminorm) such that:

The residual spectrum of each $P_{\varepsilon}$ is contained in $\{z \in \mathbb{C}:|z| \leqslant \alpha\}$.

$$
\begin{gathered}
\forall \varepsilon \in E \forall f \in V \forall n \in \mathbb{N}:\left|P_{\varepsilon}^{n} f\right|_{w} \leqslant D|f|_{w} \\
\forall \varepsilon \in E \forall f \in V \forall n \in \mathbb{N}:\left\|P_{\varepsilon}^{n} f\right\| \leqslant D \alpha^{n}\|f\|+D|f|_{w}
\end{gathered}
$$

ii. There is a monotone upper semi-continuous function $\pi: E \rightarrow[0, \infty)$ such that

$$
\lim _{\varepsilon \rightarrow 0} \pi_{\varepsilon}=0 \quad \text { and } \quad \forall f \in V \forall \varepsilon \in E:\left|P_{\varepsilon} f-P_{0} f\right|_{w} \leqslant \pi_{\varepsilon}\|f\| .
$$

An equivalent way to rewrite the last condition is:

$$
\forall \varepsilon \in E:\left\|P_{\epsilon}-P_{0}\right\| \leqslant \pi_{\varepsilon}
$$

where $\|R\|:=\sup \left\{|R f|_{w}: f \in V,\|f\| \leqslant 1\right\}$ for any linear operator $R: V \rightarrow V$.

The main result of [4] is the following theorem:

Theorem 1 Suppose that $\left(P_{\varepsilon}\right)_{\varepsilon \in E}$ is a family of linear operators on $V$ satisfying assumptions (8)(11). Fix $\delta>0$ and $r \in(\alpha, 1)$, and let $\rho:=\log \frac{r}{\alpha} / \log \frac{1}{\alpha} \in(0,1)$. Then there are constants $a=a(r)>$ $0, b=b(\delta, r)>0, c=c(\delta, r)>0$ and $d=d(\delta, r)>0$ and a neighborhood $E_{\delta, r}$ of 0 in $E$ such that for $\varepsilon \in E_{\delta, r}$ and $z \in \mathbb{C}$ with $|z|>r$ and $\operatorname{dist}\left(z, \sigma\left(P_{0}\right)\right)>\delta$,

$$
\left\|\left(z-P_{\varepsilon}\right)^{-1} f\right\| \leqslant a\|f\|+b|f|_{w} \text { for all } f \in V
$$

and

$$
\left\|\mid\left(z-P_{\varepsilon}\right)^{-1}-\left(z-P_{0}\right)^{-1}\right\| \leqslant \pi_{\varepsilon}^{\rho} \cdot\left(c\left\|\left(z-P_{0}\right)^{-1}\right\|+d\left\|\left(z-P_{0}\right)^{-1}\right\|^{2}\right) .
$$

\footnotetext{
${ }^{2}$ Assumption (3) is clearly equivalent to the seemingly stronger assumption that $\sup _{\varepsilon \in E}\left\|Q_{\varepsilon}^{n}\right\|$ decays exponentially as $n \rightarrow \infty$. The form of assumption (3) chosen here underlines however, that formula (7) can be proved as well under slightly different assumptions that allow in particular to use a weaker operator norm in (3) which is not submultiplicative, see [44, Remark 2.2].
} 
Remark 1 In [41] it is assumed that $E=[0, \infty)$, but as the parameter $\varepsilon$ enters the proofs only via the numbers $\pi_{\varepsilon}$, the same proof applies to a more general set $E$ of parameters. Similarly, in [44] it is assumed that $E$ is a closed subset of $\mathbb{R}$, but again the parameter $\varepsilon$ enters the estimates only via the derived quantities $\eta_{\varepsilon}$ and $\Delta_{\varepsilon}$ defined in section 3.1 below, so that also this result is valid for more general sets of parameters.

Remark 2 If assumptions (9) and (10) are met and if the closed unit ball of $(V,\|\cdot\|)$ is $|\cdot|_{w}$-compact, then $\sigma\left(P_{\varepsilon}\right) \backslash\{z \in \mathbb{C}:|z| \leqslant \alpha\}$ consists only of isolated eigenvalues of finite multiplicity so that assumption (8) is automatically satisfied. This is guaranteed by a strengthened version of the IonescuTulcea/Marinescu theorem (see e.g. [33]).

There are numerous situations where this remark applies:

- $V$ is a space of Hölder continuous functions on a compact metric space and $|\cdot|_{w}$ is the supremum norm. If the underlying space is not compact, weighted norms may be used; reference 33. provides many examples. In any case this setting is adapted to the study of Markov operators acting on observables and to Ruelle transfer operators, see Ruelle's monograph [51, Proposition 5.24] and [20] for a recent contribution. As this setting is less useful for studying open systems, no further details are provided here.

- $V$ is the space of functions of bounded variation on a regular domain of finite volume in $\mathbb{R}^{d}$ and $|\cdot|_{w}$ is the $L_{\mathrm{Leb}}^{1}$-seminorm. This setting is used for transfer operators of piecewise expanding maps, starting with [37, 38, 36, 52] and being continued in numerous papers, see [7] for an overview until 2000, 21] for references until 2008 and [54 for a very recent contribution to this direction. In practically all variants of this setting the passage to an open system can be described as a small perturbation of the Perron-Frobenius operator in the sense of the $\||\cdot|\|$-norm.

- More generally, $V$ is a space of distributions on a finite-dimensional smooth manifold, equipped with a suitable norm, and $|.|_{w}$ is another weaker seminorm on $V$. Starting with [14, such spaces have been applied so far to various hyperbolic or piecewise hyperbolic systems, see e.g. 32, 11, 21, 9, 10.

- $V$ is a space of distributions on $[0,1]^{\mathbb{Z}^{d}}$ which contains in particular the space of signed measures with absolutely continuous marginals of (uniformly) bounded variation (see [42, 12, 43] for details). This setting was used to study weakly coupled lattices of piecewise expanding interval maps. Inequality (10) was stated explicitly in [12, Lemma 3.4].

Remark 3 The conclusions of Theorem 1 remain valid, if assumption (8) is replaced by the following: If $\delta>0$ and $r-\alpha>0$ are sufficiently small, then the set

$$
\left\{z \in \mathbb{C}:|z|>r, \operatorname{dist}\left(z, \sigma\left(P_{0}\right)>\delta\right\}\right. \text { is connected., }
$$

see [1, Remark 6].

Now we specialize to the case where the following operator mixing condition holds:

$$
P_{0}=\varphi_{0} \otimes \nu_{0}+Q_{0} \text { as in (1) where } Q_{0} \text { has spectral radius strictly smaller than } 1 .
$$

Observe that this implies the (8) for some $\alpha \in(0,1)$. Therefore, assumptions (9) - (11) and (14) imply the validity of (11) - (3). This follows easily from Theorem 1] see [4, Corollaries 1 and 2]. It remains to show that also (44) follows: Observe first that by [41, Corollary 1],

$$
\left|\varphi_{\varepsilon}-\varphi_{0} \cdot \nu_{0}\left(\varphi_{\varepsilon}\right)\right|_{w}=\left|\varphi_{\varepsilon} \otimes \nu_{\varepsilon}\left(\varphi_{\varepsilon}\right)-\varphi_{0} \otimes \nu_{0}\left(\varphi_{\varepsilon}\right)\right|_{w} \leqslant K_{1} \pi_{\varepsilon}^{\rho} \cdot\left\|\varphi_{\varepsilon}\right\| \leqslant K_{1} K_{2} \pi_{\varepsilon}^{\rho} \cdot\left|\varphi_{\varepsilon}\right|_{w}
$$

for suitable $K_{1}, K_{2}>0$ and $\rho$ from Theorem 1, Hence

$$
\left|\varphi_{\varepsilon}\right|_{w} \cdot\left(1-K_{1} K_{2} \pi_{\varepsilon}^{\rho}\right) \leqslant\left|\varphi_{0}\right|_{w} \cdot\left|\nu_{0}\left(\varphi_{\varepsilon}\right)\right|
$$


so that $\left|\varphi_{\varepsilon}\right|_{w} \leqslant 2 \cdot\left|\varphi_{0}\right|_{w} \cdot\left|\nu_{0}\left(\varphi_{\varepsilon}\right)\right|$ when $\varepsilon \in E$ is sufficiently close to 0. Invoking [41, Corollary 1] once more, this yields

$$
\left\|\varphi_{\varepsilon}\right\| \leqslant 2 K_{2} \cdot\left|\varphi_{0}\right|_{w} \cdot\left|\nu_{0}\left(\varphi_{\varepsilon}\right)\right| \quad \text { for } \varepsilon \in E \text { sufficiently close to } 0 .
$$

Therefore one can normalize $\varphi_{\varepsilon}$ (and in consequence $\nu_{\varepsilon}$ as well) such that $\nu_{0}\left(\varphi_{\varepsilon}\right)=1$, and (4) holds for these normalized $\varphi_{\varepsilon}$ with a constant $C_{2}=2 K_{2}\left|\varphi_{0}\right|_{w}$.

\subsection{Verifying assumptions (5) and (6)}

In the remainder of this paper we restrict attention to the following dynamical setting:

REPFO (Rare event Perron-Frobenius operators) $V$ is a space of functions or distributions on a metric space $M$ that contains the constant functions, and $P_{0}$ is the Perron-Frobenius operator of a dynamical system $T: M \rightarrow M$ w.r.t. the reference measure $\nu_{0}$. It is mixing in the sense of (14). The family $\left(A_{\varepsilon}: \varepsilon \in E\right)$ is a family of subsets of $M$ which are sufficiently regular such that

i. the operators $P_{\varepsilon}, P_{\varepsilon} f=P_{0}\left(1_{M \backslash A_{\varepsilon}} f\right)$, satisfy assumptions (86) - (11) and (14),

ii. $1_{A_{\varepsilon}} \cdot f \in V$ for all $f \in V$, and

iii. $\left|\nu_{0}\left(1_{A_{\varepsilon}} f\right)\right| \cdot\left\|1_{A_{\varepsilon}} \varphi_{0}\right\| \leqslant$ const $\|f\| \cdot\left|\nu_{0}\left(1_{A_{\varepsilon}} \varphi_{0}\right)\right|$ for all $f \in V$.

Remark 4 If $V$ is a space of functions such that $\sup |f| \leqslant\|f\|$ for all $f \in V$, if $\nu_{0}$ is a measure on $M$, and if $\inf _{x \in \cup_{\varepsilon} A_{\varepsilon}} \varphi_{0}(x)>0$, then REPFO.iii is satisfied.

Remark 5 As $\nu_{0} P_{0}=\nu_{0}$, condition REPFO.iii implies (in fact, is nearly equivalent to)

$$
\left\|\nu_{0}\left(P_{0}-P_{\varepsilon}\right)\right\| \cdot\left\|\left(P_{0}-P_{\varepsilon}\right)\left(\varphi_{0}\right)\right\| \leqslant\left|\nu_{0}\left(P_{0}-P_{\varepsilon}\right)\left(\varphi_{0}\right)\right| .
$$

Let $\mu_{0}$ be the probability measure on $M$ defined by $\mu_{0}(A)=\int_{A} \varphi_{0} d \nu_{0}$. (It is the only $T$-invariant probability measure absolutely continuous w.r.t. $\nu_{0}$.) Then

$$
\mu_{0}\left(A_{\varepsilon}\right)=\nu_{0}\left(P_{0}-P_{\varepsilon}\right)\left(\varphi_{0}\right) \leqslant \text { const }\left\|\nu_{0}\left(P_{0}-P_{\varepsilon}\right)\right\| .
$$

Proposition 1 In a REPFO setting, assumptions (1)-(6) are satisfied.

Proof In the preceding section it was argued that assumptions (11) - (4) are satisfied. Next observe that for $f \in V$ with $\|f\| \leqslant 1$,

$$
\begin{array}{rlrl}
\left\|\varphi_{0}\right\| \cdot\left|\nu_{0}\left(1_{A_{\varepsilon}} f\right)\right| & =\left\|\left(\varphi_{0} \otimes \nu_{0}\right)\left(1_{A_{\varepsilon}} f\right)\right\| & \\
& =\lim _{n \rightarrow \infty}\left\|P_{0}^{n}\left(\left(P_{0}-P_{\varepsilon}\right)(f)\right)\right\| & & \text { by (3) } \\
& \leqslant D_{3}\left|\left(P_{0}-P_{\varepsilon}\right)(f)\right|_{w} & & \text { by (10) } \\
& \leqslant \pi_{\varepsilon} D_{3}\|f\| \leqslant \pi_{\varepsilon} D_{3} . & & \text { by (11) }
\end{array}
$$

Therefore,

$$
\left\|\nu_{0}\left(P_{0}-P_{\varepsilon}\right)\right\|=\sup _{\|f\| \leqslant 1}\left|\nu_{0}\left(1_{A_{\varepsilon}} f\right)\right| \leqslant \pi_{\varepsilon} \frac{D_{3}}{\left\|\varphi_{0}\right\|}
$$

and

$$
\begin{array}{rlrl}
\left\|\nu_{0}\left(P_{0}-P_{\varepsilon}\right)\right\| \cdot\left\|\left(P_{0}-P_{\varepsilon}\right) \varphi_{0}\right\| & \leqslant \text { const } \sup _{\|f\| \leqslant 1}\left|\nu_{0}\left(1_{A_{\varepsilon}} f\right)\right| \cdot\left\|1_{A_{\varepsilon}} \varphi_{0}\right\| & & \text { by (10) } \\
& \leqslant \text { const }\left|\nu_{0}\left(1_{A_{\varepsilon}} \varphi_{0}\right)\right| & & \text { by iii. } \\
& =\left|\nu_{0}\left(P_{0}-P_{\varepsilon}\right)\left(\varphi_{0}\right)\right| . &
\end{array}
$$

This proves (5) and (6).

In section 4, we discuss examples of REPFO settings. 


\section{Rare events, exponential hitting time distributions, and ex- treme value statistics}

Recall that we restrict to the REPFO setting in the remainder of this paper.

Our approach to estimate first hitting time distributions of rare events proceeds along the following well known lines: denote the first hitting time to $A_{\varepsilon}$ by $\tau_{\varepsilon}$, i.e. let $\tau_{\varepsilon}(x)=\inf \left\{i \geqslant 0: T^{i} x \in A_{\varepsilon}\right\}$ for $x \in M$. We are interested in estimating $\nu_{0}\left\{\tau_{\varepsilon} \geqslant n\right\}$ and $\mu_{0}\left\{\tau_{\varepsilon} \geqslant n\right\}$.

If $\varphi \in V$ is such that $\varphi \nu_{0}$ is a probability measure on $M$, then

$$
\begin{aligned}
\int_{\left\{\tau_{\varepsilon} \geqslant n\right\}} \varphi d \nu_{0} & =\int_{M} \prod_{i=0}^{n-1} 1_{M \backslash A_{\varepsilon}} \circ T^{i} \cdot \varphi d \nu_{0} \\
& =\int_{M} P_{0}^{n}\left(\prod_{i=0}^{n-1} 1_{M \backslash A_{\varepsilon}} \circ T^{i} \cdot \varphi\right) d \nu_{0} \\
& =\int_{M} P_{\varepsilon}^{n} \varphi d \nu_{0} \\
& =\lambda_{\varepsilon}^{n} \int_{M} \varphi_{\varepsilon} \otimes \nu_{\varepsilon}(\varphi) d \nu_{0}+\lambda_{\varepsilon}^{n} \int_{M} Q_{\varepsilon}^{n} \varphi d \nu_{0} \\
& =\lambda_{\varepsilon}^{n} \nu_{\varepsilon}(\varphi)+\mathcal{O}\left(\lambda_{\varepsilon}^{n}\left\|Q_{\varepsilon}^{n}\right\| \cdot\|\varphi\|\right)
\end{aligned}
$$

Here the constant implied by , $\mathcal{O}$ " is uniform in $n$ and $\varepsilon$. Applied with $\varphi=1$ or $\varphi=\varphi_{0}$ one obtains expressions for $\nu_{0}\left\{\tau_{\varepsilon} \geqslant n\right\}$ and $\mu_{0}\left\{\tau_{\varepsilon} \geqslant n\right\}$, respectively. To make use of this identity, we need some control of $\lambda_{\varepsilon}$ as a function of $\varepsilon$.

\subsection{The perturbation formula for the leading eigenvalue}

The main result of [44] asserts that $\lambda_{\varepsilon}=\exp \left(-\mu_{0}\left(A_{\varepsilon}\right) \theta(1+o(1))\right)$ with a constant $\theta \in(0,1]$ determined by the dynamics and a $o(1)$-term for $\varepsilon \rightarrow 0$.

For the further discussion we recall a more precise intermediate identity from [44]. Let

$$
\begin{aligned}
\eta_{\varepsilon} & :=\left\|\nu_{0}\left(P_{0}-P_{\varepsilon}\right)\right\|=\sup \left\{\int_{A_{\varepsilon}} f d \nu_{0}: f \in V,\|f\| \leqslant 1\right\} \\
\Delta_{\varepsilon} & :=\nu_{0}\left(P_{0}-P_{\varepsilon}\right)\left(\varphi_{0}\right)=\int_{A_{\varepsilon}} \varphi_{0} d \nu_{0}=\mu_{0}\left(A_{\varepsilon}\right), \\
\kappa_{N} & :=\sum_{k=N}^{\infty} \sup _{\varepsilon \in E}\left\|Q_{\varepsilon}^{k}\right\|=\mathcal{O}\left((1-\gamma)^{N}\right),
\end{aligned}
$$

where $\gamma>0$ is some lower bound for the spectral gaps of the $P_{\varepsilon}$. (It is strictly positive because of Theorem 11, see also [41, Corollary 2].) Then we have, for each $\varepsilon \in E$ with $\Delta_{\varepsilon} \neq 0$ and for each $N \in \mathbb{N}$,

$$
\frac{1-\lambda_{\varepsilon}}{\Delta_{\varepsilon}}=\left(\theta_{N, \varepsilon}+\mathcal{O}\left((1-\gamma)^{N}\right)\right)\left(1+\mathcal{O}\left(N \eta_{\varepsilon}\right)\right)
$$

with constants independent of $N$ and $\varepsilon$ [44, eq. (6.2)]3, where

$$
\theta_{N, \varepsilon}=1-\sum_{k=0}^{N-1} \lambda_{\varepsilon}^{-k} q_{k, \varepsilon} \quad \text { with } \quad q_{k, \varepsilon}=\frac{\nu_{0}\left(\left(P_{0}-P_{\varepsilon}\right) P_{\varepsilon}^{k}\left(P_{0}-P_{\varepsilon}\right)\left(\varphi_{0}\right)\right)}{\Delta_{\varepsilon}} .
$$

\footnotetext{
${ }^{3}$ If $\Delta_{\varepsilon}=0$, the numbers $q_{k, \varepsilon}$ are not well defined. Therefore the argument, given in [44 after eq.(6.2)], that $\lambda_{\varepsilon}=\lambda_{0}$ in this case, is wrong. The claim is nevertheless true, because assumption [6] implies that $\nu_{0}\left(P_{0}-P_{\varepsilon}\right)=0$ or $\left(P_{0}-P_{\varepsilon}\right)\left(\varphi_{0}\right)=0$. In both cases, $\left(\lambda_{0} / \lambda_{\varepsilon}\right)^{n}=\left(\lambda_{0} / \lambda_{\varepsilon}\right)^{n} \nu_{0}\left(\varphi_{0}\right)=\nu_{0}\left(\lambda_{\varepsilon}^{-1} P_{\varepsilon}\right)^{n}\left(\varphi_{0}\right)=\nu_{0}\left(\varphi_{\varepsilon}\right) \nu_{\varepsilon}\left(\varphi_{0}\right)+o(1) \neq 0$ when $n \rightarrow \infty$ in view of assumptions (1) - (3), so that $\lambda_{\varepsilon}=\lambda_{0}$ in view of (4).
} 
For REPFO-type $P_{\varepsilon}$ we are looking at here this simplifies, in case $\mu_{0}\left(A_{\varepsilon}\right)>0$, to

$$
q_{k, \varepsilon}=\frac{1}{\mu_{0}\left(A_{\varepsilon}\right)} \int_{A_{\varepsilon}} P_{\varepsilon}^{k} P_{0}\left(1_{A_{\varepsilon}} \varphi_{0}\right) d \nu_{0}=\frac{\mu_{0}\left(A_{\varepsilon} \cap T^{-1} A_{\varepsilon}^{c} \cap T^{-2} A_{\varepsilon}^{c} \cap \ldots \cap T^{-k} A_{\varepsilon}^{c} \cap T^{-(k+1)} A_{\varepsilon}\right)}{\mu_{0}\left(A_{\varepsilon}\right)} .
$$

Observe that $\sum_{k=0}^{\infty} q_{k, \varepsilon}=1$ for each $\varepsilon \in E$ by Kac's recurrence theorem. On the other hand, assuming that

$$
q_{k}:=\lim _{\varepsilon \rightarrow 0} q_{k, \varepsilon} \text { exists for all } k \text { and denoting } \theta:=1-\sum_{k=0}^{\infty} q_{k},
$$

this latter sum can attain any value between 0 and 1 . In any case, if these limits exist, and if we denote $\theta_{N}=1-\sum_{k=0}^{N-1} q_{k}$, we get

$$
\lim _{\varepsilon \rightarrow 0} \frac{1-\lambda_{\varepsilon}}{\mu_{0}\left(A_{\varepsilon}\right)}=\theta_{N}+\mathcal{O}\left((1-\gamma)^{N}\right) .
$$

As this is true for every $N$, we conclude, as in [44, that

$$
\lim _{\varepsilon \rightarrow 0} \frac{1-\lambda_{\varepsilon}}{\mu_{0}\left(A_{\varepsilon}\right)}=\theta:=1-\sum_{k=0}^{\infty} q_{k},
$$

i.e.

$$
\lambda_{\varepsilon}=1-\theta \mu_{0}\left(A_{\varepsilon}\right)+o\left(\mu_{0}\left(A_{\varepsilon}\right)\right)=\exp \left(-\theta \mu_{0}\left(A_{\varepsilon}\right)+o\left(\mu_{0}\left(A_{\varepsilon}\right)\right)\right) .
$$

In the next subsections we will draw some finer conclusions on the (non)-hitting probabilities $\nu_{0}\left\{\tau_{\varepsilon} \geqslant\right.$ $n\}$ and $\mu_{0}\left\{\tau_{\varepsilon} \geqslant n\right\}$ working directly with equation (17).

Remark 6 For the map $T x=2 x \bmod 1$ and $\nu_{0}$ the Lebesgue measure on $[0,1]$, formula (21) was derived by combinatorial methods in [17, Theorem 4.6.1]. In the context of Gibbs measures with a Hölder continuous potential this formula was derived also in [24, using spectral theoretic methods and ideas on exponential first return times from [34.

Remark 7 If the map $T$ is continuous, if the holes $A_{\varepsilon}$ shrink to a single point $x \in M$ and if $\mu_{0}\left(A_{\varepsilon}\right)>0$ for all $\varepsilon \neq 0$, then

i. $q_{k}=\lim _{\varepsilon \rightarrow 0} \frac{\mu_{0}\left(A_{\varepsilon} \cap T^{-(k+1)} A_{\varepsilon}\right)}{\mu_{0}\left(A_{\varepsilon}\right)}$ if $x$ is a periodic point of minimal period $k+1$,

ii. $q_{k}=0$ otherwise.

Therefore, $\theta<1$ is possible only if $x$ is a periodic point. If $x$ is a hyperbolic periodic point, then $\theta<1$, indeed.

Remark 8 Let $T:[0,1] \rightarrow[0,1]$ be a piecewise monotone interval map of Rychlik type (which always fits the REPFO setting, see section 4.1). Denote by $\nu_{0}$ the Lebesgue measure on $[0,1]$. Assume furthermore that $V$ is a finite subset of $(0,1)$, that $A_{\varepsilon}$ is the $\varepsilon$-neighbourhood of $V$, and that the invariant density $\varphi_{0}$ is continuous at each $x \in V$.

Denote by $\Pi$ the set of all pairs $(x, k) \in V \times \mathbb{N}$ such that $T^{k+1} x \in V$ and $T^{j} x \notin V$ for $j=1, \ldots, k$. It is easy to prove that

$$
\theta=1-\sum_{(x, k) \in \Pi} \frac{1}{\left|\left(T^{k}\right)^{\prime}(x)\right|} .
$$

It follows that $\theta<1$ if and only if the set $\Pi$ is nonempty. See also the related examples in [44].

Remark 9 If the holes shrink to a lower dimensional submanifold which is invariant under the dynamics, then typically $q_{0}>0$ (and hence $\theta<1$ ), see [44, section 4] for a particular example. 


\subsection{A sharp error term for exponential hitting time distributions}

Abadi [1] studied the convergence of hitting time distributions for hitting cylinder sets in a shift space over a finite alphabet $A$ under $\phi$-mixing stationary laws. He proved: Let $\mu_{0}$ be a $\phi$-mixing stationary measure on $A^{\mathbb{N}}$ with $\sum_{n} \phi(n)<\infty$. Then there are constants $C>0$ and $0<\Xi_{1}<1<\Xi_{2}<\infty$ such that for all $L \in \mathbb{N}$, cylinder sets $A=\left[a_{0} \ldots a_{L-1}\right]$ and $t \geqslant 1$, there exists $\xi_{A} \in\left[\Xi_{1}, \Xi_{2}\right]$ with the following property: for the first hitting time $\tau_{A}$ of $A$ and all $t>0$,

$$
\left|\mu_{0}\left\{\tau_{A}>\frac{t}{\xi_{A} \mu_{0}(A)}\right\}-e^{-t}\right| \leqslant C \delta(A)(t \vee 1) e^{-t}
$$

where $\delta(A)=\min _{L \leqslant N \leqslant 1 / \mu_{0}(A)}\left(N \mu_{0}(A)+\phi(N)\right)$.

An analogous result follows in the dynamical REPFO setting. One should observe, however, that Abadi assumes only a summable mixing rate whereas the REPFO setting always implies exponential decay of correlations for "regular" observables (i.e. for observables in $V$ ).

Proposition 2 Suppose that in a REPFO setting $\mu_{0}\left(A_{\varepsilon}\right)>0$ for all $\varepsilon \in E$ and that the number $\theta$ defined in (21) is strictly positive. Then there is a constant $C>0$ such that for all $\varepsilon \in E$ sufficiently close to 0 there exists $\xi_{\varepsilon}>0$ with the following property: for the first hitting time $\tau_{\varepsilon}$ of $A_{\varepsilon}$ and all $t>0$ :

$$
\left|\mu_{0}\left\{\tau_{\varepsilon} \geqslant \frac{t}{\xi_{\varepsilon} \mu_{0}\left(A_{\varepsilon}\right)}\right\}-e^{-t}\right| \leqslant C \delta_{\varepsilon}(t \vee 1) e^{-t}
$$

where $\delta_{\varepsilon}=\min _{1 \leqslant N}\left(N \eta_{\varepsilon}+\kappa_{N}\right)=\mathcal{O}\left(\eta_{\varepsilon} \log \eta_{\varepsilon}\right)$. The numbers $\xi_{\varepsilon}$ satisfy $\lim _{\varepsilon \rightarrow 0} \xi_{\varepsilon}=\theta$.

Proof Given $\varepsilon>0$, fix $N \in \mathbb{N}$ such that $N \eta_{\varepsilon}+\mathcal{O}\left(\kappa_{N}\right)$ becomes minimal, and denote this quantity by $\delta_{\varepsilon}$. Here $\mathcal{O}\left(\kappa_{N}\right)=\mathcal{O}\left((1-\gamma)^{N}\right)$ is the term from equation (17). Then $N=\mathcal{O}\left(\log \eta_{\varepsilon}\right)$ and $\delta_{\varepsilon}=\mathcal{O}\left(\eta_{\varepsilon} \log \eta_{\varepsilon}\right)$. Let $\xi_{\varepsilon}=\theta_{N, \varepsilon}+\mathcal{O}\left(\kappa_{N}\right)$. Then

$$
\lambda_{\varepsilon}=1-\mu_{0}\left(A_{\varepsilon}\right) \xi_{\varepsilon}\left(1+\mathcal{O}\left(N \eta_{\varepsilon}\right)\right)=\exp \left[-\mu_{0}\left(A_{\varepsilon}\right) \xi_{\varepsilon}\left(1+\mathcal{O}\left(\delta_{\varepsilon}\right)\right)\right]
$$

as $\mu_{0}\left(A_{\varepsilon}\right) \leqslant\left\|\varphi_{0}\right\| \cdot \eta_{\varepsilon}$.

On the other hand, $\lambda_{\varepsilon}=1-\mu_{0}\left(A_{\varepsilon}\right)(\theta+o(1))$ when $\varepsilon \rightarrow 0$ by (21). Therefore

$$
\left(\theta-\xi_{\varepsilon}\right)\left(1+\mathcal{O}\left(\delta_{\varepsilon}\right)\right)=\theta \mathcal{O}\left(\delta_{\varepsilon}\right)+o(1) \rightarrow 0
$$

so that $\lim _{\varepsilon \rightarrow 0} \xi_{\varepsilon}=\theta>0$. In particular $\xi_{\varepsilon}>0$ if $\varepsilon$ is sufficiently close to 0 .

Now let $n=\left\lfloor t /\left(\xi_{\varepsilon} \mu_{0}\left(A_{\varepsilon}\right)\right)\right\rfloor$ and assume for the moment that $n>\left|\frac{\log \eta_{\varepsilon}}{\log (1-\gamma)}\right|$. Then, in view of (15) and (16), there is some $q \in[0,1]$ such that

$$
\begin{aligned}
\mu_{0}\left\{\tau_{\varepsilon} \geqslant \frac{t}{\xi_{\varepsilon} \mu_{0}\left(A_{\varepsilon}\right)}\right\} & =\lambda_{\varepsilon}^{n+q}\left[\nu_{\varepsilon}\left(\varphi_{0}\right)+\mathcal{O}\left(\left\|Q_{\varepsilon}^{n}\right\|\right)\right] \text { from (17). } \\
& =\exp \left[-t\left(1+\mathcal{O}\left(\delta_{\varepsilon}\right)\right)\right] \cdot\left(1+\mathcal{O}\left(\eta_{\varepsilon}\right)\right)
\end{aligned}
$$

(Here we also used $\nu_{\varepsilon}\left(\varphi_{0}\right)=1+\mathcal{O}\left(\eta_{\varepsilon}\right)$, see [44, Lemma 6.1].) Therefore,

$$
\left|\mu_{0}\left\{\tau_{\varepsilon} \geqslant \frac{t}{\xi_{\varepsilon} \mu_{0}\left(A_{\varepsilon}\right)}\right\}-e^{-t}\right| \leqslant C(t \vee 1) e^{-t} \delta_{\varepsilon}
$$

(For $t \delta_{\varepsilon} \leqslant c$, where $c>0$ a suitable small constant, one can estimate by $C e^{-t} \delta_{\varepsilon}$, in the other case by $c^{-1} C t e^{-t} \delta_{\varepsilon}$.)

For $n \leqslant\left|\frac{\log \eta_{\varepsilon}}{\log (1-\gamma)}\right|$ we have the following trivial estimates:

$$
1 \geqslant \mu_{0}\left\{\tau_{\varepsilon} \geqslant \frac{t}{\xi_{\varepsilon} \mu_{0}\left(A_{\varepsilon}\right)}\right\} \geqslant \mu_{0}\left\{\tau_{\varepsilon} \geqslant n+1\right\} \geqslant 1-\sum_{i=0}^{n} \mu_{0}\left(T^{-i} A_{\varepsilon}\right)=1-(n+1) \mu_{0}\left(A_{\varepsilon}\right) \geqslant 1-\mathcal{O}\left(\delta_{\varepsilon}\right)
$$

and

$$
1 \geqslant e^{-t} \geqslant 1-t \geqslant 1-(n+1) \xi_{\varepsilon} \mu_{0}\left(A_{\varepsilon}\right) \geqslant 1-\mathcal{O}\left(\delta_{\varepsilon}\right)
$$

Hence the l.h.s. in (27) is bounded by $\mathcal{O}\left(\delta_{\varepsilon}\right)$, and as $e^{-t} \geqslant 1-\mathcal{O}\left(\delta_{\varepsilon}\right)$, this proves (27) also for $n \leqslant\left|\frac{\log \eta_{\varepsilon}}{\log (1-\gamma)}\right|$. 
Remark 10 The problem of convergence of the distributions of rescaled hitting times to an exponential without an error term as in (24) was addressed in many papers and under various assumptions, mostly for holes $A_{\varepsilon}$ that are not necessarily cylinder sets. Galves and Schmitt [30] started these investigations under an exponential $\psi$-mixing assumption. Hirata obtained a slightly weaker form of such results for Gibbs states on subshifts of finite type and for Axiom A diffeomorphisms 34 and for system with a finite generating $\varphi$ - mixing partition [35] as an important step in proving Poisson laws for these systems. For $\alpha$-mixing prcesses over a finite or countable alphabet, Abadi and Saussol [3] proved convergence of the distributions of the rescaled hitting times to an exponential without any further assumption on the mixing rate. Abadi 2 discussed in some detail the nature of the scaling factors $\xi_{A}$.

\subsection{Extreme value statistics and the extremal index}

In a series of papers, Freitas, Freitas and Todd [25, 26, 27] studied extreme value statistics for observations generated by chaotic dynamical systems and related them to first hitting time statistics. The starting point of their approach are mixing conditions in the tradition of Leadbetter [45, 46] that are adapted to the problem of studying extremal events. They show that these conditions are in particular satisfied if the underlying system is a (topologically mixing) one-dimensional map of Rychlik type [52] and $\nu_{0}$ and $\mu_{0}$ are the associated conformal resp. invariant measure. Here we show how some of their results can be derived (and slightly extended) from our formula (21).

We begin recalling the approach to extreme value laws taken in [25, 26, 27. Suppose that $X$ : $M \rightarrow \mathbb{R}$ is a continuous function. Let $z_{\max }:=\sup \left\{z \in \mathbb{R}: \nu_{0}(X \geqslant z)>0\right\}$ and assume that for some $z_{0}<z_{\max }$ all sets $V_{z}:=\{X>z\}$ for $z \in\left[z_{0}, z_{\max }\right]$ satisfy the assumptions on the sets $A_{\varepsilon}$ from above. Suppose for simplicity that $\nu_{0}\left(V_{z_{\max }}\right)=0$. Fix $t>0$ and determine real numbers $z_{n} \in\left[z_{0}, z_{\max }\right]$ such that $\lim _{n \rightarrow \infty} n \mu_{0}\left\{X>z_{n}\right\}=t$. Consider the process $X_{n}=X \circ T^{n}$ on the probability space $\left(M, \mathcal{B}, \nu_{0}\right)$. Then $\max \left(X_{0}(\omega), \ldots, X_{n-1}(\omega)\right) \leqslant z_{n}$ if and only if $T^{i} \omega \in M \backslash V_{z_{n}}$ for $i=0, \ldots, n-1$. Denote $\varepsilon=\varepsilon_{n}:=z_{\max }-z_{n}$. Then equations (15) and (22) imply the following extreme value law

$$
\begin{aligned}
\nu_{0}\left\{\max \left(X_{0}, \ldots, X_{n-1}\right) \leqslant z_{n}\right\} & =\nu_{0}\left\{\omega \in M: T^{i} \omega \notin V_{z_{n}} \text { for } i=0, \ldots, n-1\right\} \\
& =\nu_{0}\left\{\tau_{\varepsilon} \geqslant n\right\} \\
& =\exp (-t \theta(1+o(1)))
\end{aligned}
$$

in the limit $n \rightarrow \infty$, where also the fact that $\lim _{\varepsilon \rightarrow 0} \nu_{\varepsilon}(1)=\nu_{0}(1)=1$ (see [41]) is used. In this context the constant $\theta$ defined in (20) is called the extremal index. It follows from Remark 7 that in

case $V_{z_{\max }}=\{x\}$ is a singleton, $\theta<1$ is possible only if $x$ is a periodic point. In view of the results collected in section 4 , this answers a question by Freitas, Freitas and Todd from the introduction of [26.

\section{A review of open systems to which the eigenvalue pertur- bation formula applies}

In this section we discuss some classes of open systems of type REPFO. The results of section 3 apply to these systems.

\subsection{Piecewise monotone interval maps of Rychlik type}

Rychlik [52] provided a very elegant and useful scheme to treat piecewise monotone one-dimensional maps $T:[0,1] \rightarrow[0,1]$. The ingredients of his approach are a non-atomic probability measure $\nu_{0}$ on $[0,1]$ with full support and a function $g:[0,1] \rightarrow[0,+\infty)$ of bounded variation. It is assumed that

a) there is an open set $U \subset[0,1]$ with $\nu_{0}(U)=1$ such that for each connected component $J$ of $U$, the restriction $T_{\mid J}$ is a homeomorphism from $J$ onto $T J$ (denote the collection of open components of $U$ by $\beta$ ),

b) $g_{\mid S} \equiv 0$ where $S:=[0,1] \backslash U$, 
c) $\vartheta:=\lim _{n \rightarrow \infty}\left\|g_{n}\right\|_{\infty}^{1 / n}<1$ where $g_{n}=g \cdot g \circ T \cdot \ldots \cdot g \circ T^{n-1}$, and

d) $\nu_{0}(P f)=\nu_{0}(f)$ for each bounded measurable $f:[0,1] \rightarrow \mathbb{R}$ where

$$
P f(x)=\sum_{y \in T^{-1} x} g(y) f(y) .
$$

Denote by $B V$ the space of functions of bounded variation on $[0,1]$ and define the seminorm $|f|_{w}=$ $\int|f| d \nu_{0}$ and the norm $\|f\|=\operatorname{Var}(f)+|f|_{w}$ on $B V$. Then assumption d) implies (9) for $\varepsilon=0$, and Proposition 1 of [52] shows that

$$
\forall \alpha \in(\vartheta, 1) \exists C>0 \forall f \in B V \forall n \in \mathbb{N}: \sum_{B \in \beta^{n}} \operatorname{Var}\left(P^{n}\left(f 1_{B}\right)\right) \leqslant C \kappa^{n} \operatorname{Var}(f)+C \int|f| d \nu_{0}
$$

where $\beta^{n}=\beta \vee T^{-1} \beta \vee \ldots \vee T^{-(n-1)} \beta$. This is (10) for $\varepsilon=0$. Finally, (8) for $\varepsilon=0$ follows from [52, Theorem 1].

Let $z \in(0,1)$ and consider intervals $A_{\varepsilon}=(z-\varepsilon, z+\varepsilon) \subset(0,1)$. If we replace $S$ by $S \cup\{z\}$, all of the above assumptions a) $-\mathrm{d}$ ) remain valid. So w.l.o.g. $z \in S$. The rare event PF operators $P_{\varepsilon}$ satisfy

$$
\left|P_{\varepsilon}^{n} f\right|_{w}=\int_{\bigcap_{k=0}^{n-1} T^{-k}\left([0,1\} \backslash A_{\varepsilon}\right)}|f| d \nu_{0} \leqslant \int|f| d \nu_{0}=|f|_{w},
$$

and, observing that $B \cap \bigcap_{k=0}^{n-1} T^{-k}\left([0,1\} \backslash A_{\varepsilon}\right)$ is a subinterval (possibly empty) of $B$ for each $B \in \beta^{n}$, also

$$
\operatorname{Var}\left(P_{\varepsilon}^{n} f\right) \leqslant \sum_{B \in \beta^{n}} \operatorname{Var}\left(P_{\varepsilon}^{n}\left(f 1_{B}\right)\right) \leqslant \sum_{B \in \beta^{n}} \operatorname{Var}\left(P^{n}\left(f 1_{B}\right)\right) \leqslant C \kappa^{n} \operatorname{Var}(f)+C|f|_{w} .
$$

The uniform estimates (30) and (31) show that (18) - (10) are satisfied for all $\varepsilon>0$. We turn to (11):

$$
\left|P_{\varepsilon} f-P f\right|_{w} \leqslant \int_{A_{\varepsilon}}|f| d \nu_{0} \leqslant \nu_{0}\left(A_{\varepsilon}\right)\|f\|_{\infty} \leqslant \nu_{0}\left(A_{\varepsilon}\right)\|f\| .
$$

So $\tau_{\varepsilon}=\nu_{0}\left(A_{\varepsilon}\right) \searrow 0$ as $\varepsilon \rightarrow 0$, and this is (11). Thus assumption REPFO.i is verified.

Assume now that $T$ is mixing in the sense of (14) with unique invariant density $\varphi_{0}$. As $\varphi_{0}$ is of bounded variation, the following extra assumption is not very restrictive:

Positivity assumption: $\delta:=\lim _{\varepsilon \rightarrow 0} \max \left\{\inf _{(z-\varepsilon, z)} \varphi_{0}, \inf _{(z, z+\varepsilon)} \varphi_{0}\right\}>0$.

Indeed, if $T$ has only finitely many monotone branches, Theorem 8.2.3 in [15] shows that this assumption holds if $z$ belongs to the support of the invariant measure $f_{0} \nu_{0}$.

Under the positivity assumption,

$$
\left\|1_{A_{\varepsilon}} f\right\|=\int_{A_{\varepsilon}}|f| d \nu_{0}+\operatorname{Var}\left(1_{A_{\varepsilon}} f\right) \leqslant|f|_{w}+2\|f\|_{\infty}+\operatorname{Var}(f) \leqslant 3\|f\|
$$

and

$$
\left|\nu_{0}\left(1_{A_{\varepsilon}} f\right)\right| \cdot\left\|1_{A_{\varepsilon}} \varphi_{0}\right\| \leqslant \nu_{0}\left(A_{\varepsilon}\right)\|f\|_{\infty} \cdot 3\left\|\varphi_{0}\right\| \leqslant 4 \delta^{-1}\left\|\varphi_{0}\right\| \cdot \nu_{0}\left(1_{A_{\varepsilon}} \varphi_{0}\right) \cdot\|f\|
$$

for all suficiently small $\varepsilon>0$. These two estimates yield REPFO.ii and iii.

Remark 11 The class of Rychlik maps contains the classes of maps considered in [4] and [48]. Freitas et al. 26] proved the validity of an extreme value law (28) for Rychlik maps along the classical probabilistic lines initiated by Leadbetter [45, 46]. Exponential statistics for Rychlik maps around $\mu_{0}$-a.e. point $z$ were already derived in [16].

See also Remark 8 for an explicit formula for $\theta$. 


\subsection{Piecewise expanding maps in higher dimensions}

For piecewise expanding maps in higher dimensions, the classical BV-space is the first candidate for a suitable function space $V$ 31, 37, 54. For the present purpose, however, the quasi Hölder spaces of Blank [13] and Saussol [53] are more suited, because, in contrast to classical BV-spaces, these spaces are algebras and their norms control the $L^{\infty}$-norm [53, Proposition 3.4].

Without going into details, we consider a piecewise $C^{1+\alpha}$ map on a compact set $\Omega \subset \mathbb{R}^{N}$ for which expansion wins over the effects of discontinuities in the sense of [53, condition (PE5)]. The corresponding PF operator $P$ satisfies (8) - (10) for $\varepsilon=0$ in view of [53, Lemma 4.1 and Theorem 5.1]. Fix a point $z$ in the interior of the domain $U_{i}$ of one of the branches of $T$, and denote by $A_{\varepsilon}$ the $\varepsilon$-neighborhood of $z$ (w.r.t. some $\ell_{p}$-norm in $\mathbb{R}^{N}$ ). Then the corresponding rare event PF operators $P_{\varepsilon}$ satisfy the same estimates that $P$ satisfies if the domain $U_{i}$ is replaced by $U_{i} \backslash A_{\varepsilon}$ (for sufficiently small $\varepsilon$ ). This modification of $U_{i}$ increases the constant $G$ in (PE5) of $[53]$ by at most const $\varepsilon^{N-1}$, and it follows from the explicit expressions for the constants in (10) that are given in the proof of Lemma 4.1 in [53, that (8) - (10) are indeed valid for all sufficiently small $\varepsilon>0$. Assumption (11) is satisfied because $\|f\|_{\infty} \leqslant$ const $\|f\|$ where $\|$.$\| is Saussol's quasi Hölder norm. This is REPFO.i. As$ $\left\|1_{A_{\varepsilon}}\right\|$ is of the order of $\varepsilon^{N-1}$, REPFO.ii follows from $\left\|1_{A_{\varepsilon}} f\right\| \leqslant$ const $\left\|1_{A_{\varepsilon}}\right\| \cdot\|f\|$, see [53. Proposition 3.4]. Under the additional positivity assumption $\delta:=\lim _{\varepsilon \rightarrow 0} \inf _{A_{\varepsilon}} \varphi_{0}>0$, condition REPFO.iii follows immediately from $\|f\|_{\infty} \leqslant$ const $\|f\|$.

In higher dimensional systems, holes that do not shrink to a point may occur quite naturally, for example when $A_{\varepsilon}$ is defined as the event that a state vector has two nearly (i.e.up to $\varepsilon$ ) identical coordinates. A simple example of this type, namely a direct product of a piecewise expanding interval map $T$ with itself, is discussed in [19, where exponential hitting time statistics are proved (as a corollary to a Poissonian limit law) and where a (complicated) formula for the exponent of the limit distribution is derived also in the case where only some power $T^{m}$ of the map is uniformly expanding. In [44, section 4] this formula is rederived from spectral perturbation theory when $T$ itself is expanding. Indeed, for $m>1$, too, this formula can be derived from (21) by a straightforward, though lengthy calculation. In the same reference also a modification of the product system by some weak coupling is discussed.

\subsection{Gibbs measures on subshifts of finite type}

Ferguson and Pollicott 24] study escape rates for Gibbs measures with Hölder continuous potentials on subshifts of finite type. They obtain formula (21) for suitable holes (not just cylinder sets) in this context and use it to derive from that the corresponding formula for the escape from conformal repellors relative to equilibrium states, and also a related formula for the Hausdorff dimension of the set of points on the repellor that never enter the hole. For deriving formula (21) they produce a function space and two norms fitting the REPFO setting.

\subsection{Topological entropy of perturbations of shifts of finite type}

If a subshift of finite type over a finite alphabet is perturbed by deleting one or several blocks and if these blocks have small mass under the measure $\mu_{0}$ of maximal entropy of the unperturbed subshift, the topological entropy of the systems drops by a small amount. As the topological entropy is the logarithm of the leading eigenvalue of the corresponding transfer operator with constant weight 1 , the amount by which the entropy drops down is controlled by formula (21) when it is applied to the operators normalized such that the unperturbed operator has leading eigenvalue 1.

Lind [47, Theorem 3] provides upper and lower bounds on the entropy difference when a single (long) block is discarded. These estimates can be made more precise using formula (21). Details of this in two slightly different settings can be found in [44, section 5.2] and in [24, Corollary 5.4].

\footnotetext{
${ }^{4}$ The constant there is denoted $G\left(\varepsilon, \varepsilon_{0}\right)$; note that our $\varepsilon$ (size of the hole) and the $\varepsilon$ in $G\left(\varepsilon, \varepsilon_{0}\right)$ are unrelated.
} 


\subsection{Further candidates}

The investigation of statistical properties of dynamical systems via the spectral properties of their associated Perron Frobenius operators is currently a very active area of research. Some of the available results apply to piecewise smooth systems and there is good hope that these systems are of REPFO type. Here we discuss briefly the situation for piecewise hyperbolic maps and for coupled map lattices of piecewise expanding $1 \mathrm{D}$ maps.

Piecewise hyperbolic maps: Demers and Liverani 21] studied Perron-Frobenius operators for a broad class of piecewise hyperbolic maps on two-dimensional compact Riemannian manifolds. They provide a normed space $(\mathcal{B},\|\|$.$) of distributions and prove in [21, Lemmas 3.5$ and 6.5, Proposition 6.6] that assumptions (8) - (11) hold for many reasonably regular holes $A_{\varepsilon}$. In particular this is true for disks of radius $\varepsilon$ when $\varepsilon$ is sufficiently small (so that the curvature of $\partial A_{\varepsilon}$ is large, see [21, Remark 2.16]). Hence REPFO.i is satisfied. A first step towards a proof of REPFO.iii is the observation that $\left|\nu_{0}\left(1_{A_{\varepsilon}} f\right)\right| \leqslant \varepsilon^{1+\alpha}\|f\|_{s} \leqslant\|f\|$ for all $f \in V$ according to the definition of the stable norm $\|\cdot\|_{s}$ and the strong norm $\|$.$\| in [21, eq. (2.3)and (2.5)]. Therefore, it mainly remains to show that$ $\varepsilon^{1+\alpha}\left\|1_{A_{\varepsilon}} \varphi_{0}\right\| \leqslant \nu_{0}\left(1_{A_{\varepsilon}} \varphi_{0}\right)$.

Baladi and Gouëzel [9, 10] studied the Perron Frobenius operator of piecewise hyperbolic systems in any dimension on certain classes of Triebel spaces which they chose such that multiplication by a regular set, e.g. a convex set, acts as a bounded linear operator. This indicates that also their systems might fit into the REPFO setting.

Coupled map lattice of piecewise expanding interval maps: Coupled map lattices (over $\mathbb{Z}^{d}$ ) of piecewise expanding interval maps were studied in [42, 43] using a spectral theoretic approach. Here $V$ is a space of distributions on $[0,1]^{\mathbb{Z}^{d}}$ that contains in particular all probability measures whose finite dimensional marginals have densities of bounded variation with uniform bounds on the variation of all these marginal densities in all coordinate directions. While 42 treats only coupling mechanisms which are uniformly weak on the whole phase space, the short note [43] deals with cases where strong (and discontinuous) coupling effects are allowed on small parts of the phase space. The estimates used in this context should be helpful also in attempting to prove REPFO.i-iii.

\section{References}

[1] M. Abadi. Sharp error terms and neccessary conditions for exponential hitting times in mixing processes. The Annals of Probability, 32(1A):243-264, 2004.

[2] M. Abadi. Hitting, returning and the short correlation function. Bull. Braz. Math. Soc. (N.S.), 37:593-609, 2006.

[3] M. Abadi and B. Saussol. Hitting and returning into rare events for all alpha-mixing processes. Stochastic Processes and their Applications, 121:314-323, 2011.

[4] W. Bahsoun and C. Bose. Quasi-invariant measures, escape rates and the effect of the hole. Discrete and Continuous Dynamical Systems, 27:1107-1121, 2010.

[5] W. Bahsoun and C. Bose. Invariant densities and escape rates: Rigorous and computable approximations in the $L^{\infty}$-norm. Nonlinear Analysis, 74:4481-4495, 2011.

[6] W. Bahsoun and S. Vaienti. Metastability of certain intermittent maps. Arxiv preprint arXiv:1105.0538, 2011.

[7] V. Baladi. Positive transfer operators and decay of correlations, volume 16 of Advanced Series in Nonlinear Dynamics. World Scientific, 2000.

[8] V. Baladi. On the susceptibility function of piecewise expanding interval maps. Communications in Mathematical Physics, 275:839-859, 2007. 
[9] V. Baladi and S. Gouëzel. Good Banach spaces for piecewise hyperbolic maps via interpolation. Annales de l'Institut Henri Poincaré (C) Non Linear Analysis, 2009.

[10] V. Baladi and S. Gouëzel. Banach spaces for piecewise cone-hyperbolic maps. Journal of Modern Dynamics, 4:91-137, 2010.

[11] V. Baladi and M. Tsujii. Anisotropic Hölder and Sobolev spaces for hyperbolic diffeomorphisms. Annales de l'Institut Fourier, 57:127-154, 2007.

[12] J.-B. Bardet, S. Gouëzel, and G. Keller. Limit theorems for coupled interval maps. Stochastics and Dynamics, 7:17-36, 2007.

[13] M. Blank. Stochastic properties of deterministic dynamical systems. Soviet Scientific Reviews, Section C: Mathematical Physics Reviews, 6:243-271, 1987.

[14] M. Blank, G. Keller, and C. Liverani. Ruelle-Perron-Frobenius spectrum for Anosov maps. Nonlinearity, 15:1-69, 2002.

[15] A. Boyarsky and P. Góra. Laws of Chaos. Probability and its Applications. Birkhäuser, 1997.

[16] H. Bruin, B. Saussol, S. Troubetzkoy, and S. Vaienti. Return time statistics via inducing. Ergodic Theory \& Dynamical Systems, 23:991-1013, 2003.

[17] L. Bunimovich and A. Yurchenko. Where to place a hole to achieve a maximal escape rate. Israel Journal of Mathematics, 182:229-252, 2011.

[18] Z. Coelho. Asymptotic laws for symbolic dynamical systems. In F. Blanchard, A. Maass, and A. Nogueira, editors, Topics in Symbolic Dynamics and Applications, pages 123-165. Cambridge University Press, 2000.

[19] Z. Coelho and P. Collet. Asymptotic limit law for the close approach of two trajectories of expanding maps of the circle. Probability Theory and Related Fields, 99:237-250, 1994.

[20] V. Cyr and O. Sarig. Spectral gap and transience for Ruelle operators on countable Markov shifts. Communications in Mathematical Physics, 292:637-666, 2009.

[21] M. Demers and C. Liverani. Stability of statistical properties in two-dimensional piecewise hyperbolic maps. Transactions of the Amer.Math. Soc., 360:4777-4814, 2008.

[22] M. Demers, P. Wright, and L.-S. Young. Entropy, Lyapunov exponents and escape rates in open systems. Arxiv preprint arXiv:1107.2609, 2011.

[23] M. Demers and L.-S. Young. Escape rates and conditionally invariant measures. Nonlinearity, 19:377-397, 2006.

[24] A. Ferguson and M. Pollicott. Escape rates for Gibbs measures. To appear in Ergodic Theory and Dynamical Systems, DOI: 10.1017/S0143385711000058. .

[25] A. Freitas, J. Freitas, and M. Todd. Hitting time statistics and extreme value theory. Probability Theory and Related Fields, 147:675-710, 2009.

[26] A. Freitas, J. Freitas, and M. Todd. Extremal index, hitting time statistics and periodicity. Arxiv preprint arXiv:1008.1350v1, 2010.

[27] A. Freitas, J. Freitas, and M. Todd. Extreme value laws in dynamical systems for non-smooth observations. Journal of Statistical Physics, 142:108-126, 2010.

[28] G. Froyland, R. Murray, and O. Stancevic. Spectral degeneracy and escape dynamics for intermittent maps with a hole. Nonlinearity, 24:2435-2463, 2011.

[29] G. Froyland and O. Stancevic. Escape rates and Perron-Frobenius operators: Open and closed dynamical systems. Discrete and Continuous Dynamical Systems - Series B, 14:457-472, 2010. 
[30] A. Galves and B. Schmitt. Occurence time of rare events for mixing dynamical systems. Annales de l'IHP Physique théorique, 52:267-281, 1990.

[31] P. Góra and A. Boyarsky. Absolutely continuous invariant measures for piecewise $C^{2}$ transformations in $\mathbb{R}^{N}$. Israel Journal of Mathematics, 67:272-286, 1989.

[32] S. Gouëzel and C. Liverani. Banach spaces adapted to Anosov systems. Ergodic Theory and Dynamical Systems, 26:189-217, 2006.

[33] H. Hennion and L. Hervé. Limit Theorems for Markov Chains and Stochastic Properties of Dynamical Systems by Quasi-Compactness, volume 1766 of Lecture Notes in Mathematics. Springer, 2001.

[34] M. Hirata. Poisson law for Axiom A diffeomorphisms. Ergodic Theory 8 Dynamical Systems, 13:533-556, 1993.

[35] M. Hirata. Poisson law for the return times of some chaotic dynamical systems. Progress of Theor. Physics Suppl., 116:351-358, 1994.

[36] F. Hofbauer and G. Keller. Ergodic properties of invariant measures for piecewise monotonic transformations. Mathematische Zeitschrift, 180(1):119-140, March 1982.

[37] G. Keller. Ergodicité et mesures invariantes pour les transformations dilatantes par morceaux d'une région bornée du plan. C. R. Acad. Sci. Paris, Série A, 289:625-627, 1979.

[38] G. Keller. Un théorème de la limite centrale pour une classe de transformations monotones par morceaux. C. R. Acad. Sci. Paris, Série A, 291:155-158, 1980.

[39] G. Keller. On the rate of convergence to equilibrium in one-dimensional systems. Communications in Mathematical Physics, 96:181-193, 1984.

[40] G. Keller. Markov extensions, zeta functions, and Fredholm theory for piecewise invertible dynamical systems. Transactions Amer. Math. Soc., 314:433-497, 1989.

[41] G. Keller and C. Liverani. Stability of the spectrum for transfer operators. Ann. Scuola Norm. Sup. Pisa Cl. Sci.(4), 28:141-152, 1999.

[42] G. Keller and C. Liverani. Uniqueness of the SRB measure for piecewise expanding weakly coupled map lattices in any dimension. Communications in Mathematical Physics, 262:33-50, 2006.

[43] G. Keller and C. Liverani. Map lattices coupled by collisions. Communications in Mathematical Physics, 597:591-597, 2009.

[44] G. Keller and C. Liverani. Rare events, escape rates and quasistationarity: Some exact formulae. Journal of Statistical Physics, 135:519-534, 2009.

[45] M.R. Leadbetter. On extreme values in stationary sequences. Z. Wahrscheinlichkeitstheorie und Verw. Gebiete, 28:289-303, 1973.

[46] M.R. Leadbetter. Extremes and local dependence in stationary sequences. Z. Wahrscheinlichkeitstheorie und Verw. Gebiete, 65:291-306, 1983.

[47] D. Lind. Perturbations of shifts of finite type. SIAM Journal on Discrete Mathematics, 2:350-365, 1989.

[48] C. Liverani and V. Maume-Deschamps. Lasota-Yorke maps with holes: conditionally invariant probability measures and invariant probability measures on the survivor set. Annales de l'Institut Henri Poincaré (B) Probability and Statistics, 39:385-412, 2003. 
[49] G. Pianigiani. Conditionally invariant measures and exponential decay. J. Math. Analysis Appl., 82:75-88, 1981.

[50] G. Pianigiani and J.A. Yorke. Expanding maps on sets which are almost invariant: decay and chaos. Transactions Amer. Math. Soc., 252:351-366, 1979.

[51] D. Ruelle. Thermodynamic Formalism, volume 5 of Encyclopedia of Mathematics and its Applications. Addison-Wesley, 1978.

[52] M. Rychlik. Bounded variation and invariant measures. Studia Mathematica, 76:69-80, 1983.

[53] B. Saussol. Absolutely continuous invariant measures for multidimensional expanding maps. Israel Journal of Mathematics, 116:223-248, 2000.

[54] D. Thomine. A spectral gap for transfer operators of piecewise expanding maps. Discrete and Continuous Dynamical Systems, 30:917-944, 2011. 